\title{
The Isometry Group of an RCD* Space is Lie
}

\author{
Gerardo $\operatorname{Sosa}^{1}$ (D)
}

Received: 15 April 2017 / Accepted: 16 September 2017 / Published online: 12 October 2017

(C) The Author(s) 2017. This article is an open access publication

\begin{abstract}
We give necessary and sufficient conditions that show that both the group of isometries and the group of measure-preserving isometries are Lie groups for a large class of metric measure spaces. In addition we study, among other examples, whether spaces having a generalized lower Ricci curvature bound fulfill these requirements. The conditions are satisfied by RCD*-spaces and, under extra assumptions, by CD-spaces, $C D^{*}$-spaces, and MCP-spaces. However, we show that the MCP-condition by itself is not enough to guarantee a smooth behavior of these automorphism groups.
\end{abstract}

Keywords Metric spaces · Group actions · Lie groups · Ricci curvature · Synthetic Ricci curvature $\cdot$ Optimal transport

Mathematics Subject Classifications (2010) 51Fxx $\cdot 22 \mathrm{Exx} \cdot 53 \mathrm{C} 21$

\section{Introduction}

We give necessary and sufficient conditions to assure that the group of isometries and the group of measure-preserving isometries are Lie groups for a certain class of metric measure spaces. Additionally we analyze spaces that fulfill these assumptions, for example spaces that satisfy a particular curvature-dimension condition. Such is the case of RCD*-spaces, and of $C D / C D^{*}$-spaces, and MCP-spaces satisfying mild hypotheses. More generally we show that spaces with good optimal transport properties meet as well the hypotheses.

In certain classes of spaces the full group of isometries, $\operatorname{ISO}(X)$, is known to be a Lie group. For example, Myers and Steenrod proved this fact for Riemannian Manifolds in [26], Fukaya and Yamaguchi for Alexandrov spaces with curvature bounded by above and by

Gerardo Sosa

gsosa@mis.mpg.de

1 Max Planck Institute for Mathematics in the Sciences, Leipzig 04103, Germany 
below in $[12,36]$, and Cheeger, Colding, and Naber in the case of Ricci Limit spaces in $[4,6]$. In contrast, there exist metric spaces for which $\operatorname{ISO}(X)$ is not a Lie group, see for instance Examples 5.1 and 5.2.

To state the results let $(X, \mathrm{~d})$ be a complete, separable metric space and assume that $\mathfrak{m}$ is a fully supported Borel measure on $X$ which is finite on every bounded set. We call the triple $(X, \mathrm{~d}, \mathfrak{m})$ a metric measure space, mms for short, and let $\mathrm{G} \in\left\{\operatorname{ISO}(X), \operatorname{ISO}_{\mathfrak{m}}(X)\right\}$ denote either the group of isometries or the group of measure-preserving isometries of $X$. The fixed point set of an isomorphism $f \in \mathrm{G}$ is denoted by Fix $(f)$.

Theorem 1.1 (mms with smooth automorphism groups) Let $(X, \mathrm{~d}, \mathfrak{m})$ be a locally compact $\mathrm{mms}$ where every closed ball coincides with the closure of its respective open ball. Assume that $X$ has $\mathfrak{m}$-a.e. unique tangent cones which are Euclidean. Then $\mathrm{G}$ is a Lie group if and only if:

(a) There exist $x \in X$ and constants $0<s, 0<\mathrm{FIX}<\mathfrak{m}\left(B_{s}(x)\right)$ such that for every $(\mathbb{I} \neq) g \in \mathrm{G}$

$$
\mathfrak{m}\left(\operatorname{Fix}(g) \cap B_{s}(x)\right)<\operatorname{FIX} .
$$

Moreover if $\mathrm{ISO}(X)$ is a Lie group then $\mathrm{ISO}_{\mathfrak{m}}(X)$ is so as well.

Furthermore, by a theorem of van Danzig and van der Waerden [8] and Lemma 4.5 we can conclude that $\operatorname{ISO}(X)$ and $\operatorname{ISO}_{\mathfrak{m}}(X)$ are compact if $X$ is compact.

As a matter of fact Theorem 1.1 remains valid when considering mms in which the tangent cones are well-behaved $\mathfrak{m}$-almost everywhere yet might fail to be Euclidean, see Remark 4.6 for the precise statement. For example, such situation arises when $X$ has $\mathfrak{m}$ almost everywhere unique tangent cones and the set of spaces appearing as unique tangents is a finite union of Euclidean spaces, normed spaces, and Carnot groups. Accordingly, we are also able to study spaces with Finsler-like geometries rather than only Riemannian ones. We recall a result of relevance in this direction due to Le Donne [7] that states that geodesic spaces with a doubling measure that have $\mathfrak{m}$-a.e. unique tangents have $\mathfrak{m}$-a.e. Carnot groups as tangents.

In view of the remark the following are examples of mms satisfying condition (a) and the hypotheses of Theorem 1.1: Weighted Riemannian manifolds and Finsler manifolds with the Holmes-Thompson or Busemann-Hausdorff volume measure; correspondingly, Alexandrov spaces with curvature bounded below and a class of their Finsler counterpart, Busemann concave spaces [19], both endowed with the Hausdorff measure. One may ask whether these hypotheses are also granted by weaker curvature bounds. For example by curvaturedimension conditions which use optimal mass transport theory to generalize the notion of a lower Ricci curvature bound to metric measure spaces. These conditions are variations that developed from an initial condition introduced independently by Lott and Villani and by Sturm in [24, 32, 33]. Important contributors to these developments are L. Ambrosio, K. Bacher, M. Erbar, K. Kuwada, N. Gigli, A. Mondino, T. Rajala, G. Savaré, and K.T. Sturm. For a historical recount one can consult for example the introductions of [9, 25].

We consider spaces satisfying the Riemannian curvature-dimension condition, the (reduced) curvature-dimension condition, and the measure contraction property, and write RCD*, $\left(C D^{*}\right) C D$, and MCP, for short. The relation between these spaces can be written as $\mathrm{RCD}^{*}$ spaces $\subsetneq(\mathrm{CD}) \mathrm{CD}^{*}$ spaces $\subsetneq$ MCPspaces, where all inclusions are proper. It turns out that RCD*-spaces have smooth isomorphism groups.

Corollary 1.2 (Automorphisms of RCD*-spaces) Let $K \in \mathbb{R}, N \in[1, \infty)$, and $(X, \mathrm{~d}, \mathfrak{m})$ be an $\operatorname{RCD}^{*}(K, N)$-space. Then the groups $\operatorname{ISO}_{\mathfrak{m}}(X)$ and $\mathrm{ISO}(X)$ are Lie groups. 
Examples of RCD*-spaces are Alexandrov and Ricci limit spaces with the Hausdorff measure, generalized cone constructions over RCD*-spaces, and limits of weighted manifolds with a lower bound on the Bakry-Emery Ricci tensor [20, 24, 28, 32, 33]. However, it is not known whether the class of RCD*-spaces is strictly bigger than that of weighted Ricci limit spaces.

We study as well spaces satisfying different curvature-dimension conditions. Recall that an mms is essentially non-branching and satisfies the (CD-)CD*-condition if and only if it satisfies the strong $(C D-) C D^{*}$-condition; for these results and definitions see $[5,30]$.

Corollary 1.3 (Automorphisms of CD-, CD*-, and MCP-spaces) Let $K$ and $N$ be as above. The groups $\operatorname{ISO}(X)$ and $\operatorname{ISO}_{\mathfrak{m}}(X)$ are Lie groups for strong $\mathrm{CD} / \mathrm{CD}^{*}(K, N)$-spaces and essentially non-branching $\operatorname{MCP}(K, N)$-spaces that have $\mathfrak{m}$-a.e. Euclidean tangents.

The CD-, CD*-, and MCP-conditions allow for non-Riemannian geometries which include, but are not restricted to, Finsler manifolds. Consistently with the remark following Theorem 1.1, the above corollary is still valid in spaces with these kind of metrics granted that the tangent cones are well-behaved, see Remark 4.6. For example, any corank 1 Carnot group of dimension $(k+1)$ equipped with a left-invariant measure is an essentially nonbranching MCP-space with unique non-Euclidean tangents by Rizzi [29], it follows from our results that their automorphism groups are Lie groups.

The above corollaries are particular examples of a larger class of spaces for which condition (a) holds.

Theorem 1.4 Let $(X, \mathrm{~d}, \mathfrak{m})$ be a locally compact, length metric measure space. Assume that for all probability measures $\mu_{0}(\ll \mathfrak{m}), \mu_{1} \in \mathcal{P}_{2}(X)$ any optimal transport plan between $\mu_{0}$ and $\mu_{1}$ is induced by a map. Then condition (a) is satisfied.

In particular, if $X$ has well-behaved tangent cones then $\mathrm{G}$ is a Lie group.

Indeed, from the results of Gigli-Rajala-Sturm [16], and Cavalletti-Mondino [5] it's known that transport plans starting from absolutely continuous measures in RCD*-, strong CD/CD*-, and essentially non-branching MCP-spaces are given by maps. Moreover it was proved in Mondino-Naber [25] that $\operatorname{RCD}^{*}(K, N)$-spaces have $\mathfrak{m}$-a.e. unique Euclidean tangents for finite $N$.

On the other hand we show that a weak curvature-dimension condition by itself might not be restrictive enough to guarantee smooth automorphism groups.

Proposition 1.5 There exists an $\operatorname{MCP}(2,3)$-space for which neither $\operatorname{ISO}_{\mathfrak{m}}(X)$ nor $\operatorname{ISO}(X)$ are Lie groups.

We describe now the idea of the proof of the main theorem. A remarkable result of Gleason and Yamabe in the early 1950's asserts that a locally compact, topological group is not a Lie group if and only if every neighborhood of the identity has a non-trivial subgroup. ${ }^{1}$ If a group has this property we say that it has the small subgroup property, ssp, for short. The strategy is to show the contrapositive statement in Theorem 1.1. Supposing that $\operatorname{ISO}(X)$ is not a Lie group, by using a blow up argument, we show that the assumption of the

\footnotetext{
${ }^{1}$ See Theorem 2.6 and the remarks below.
} 
$\mathfrak{m}$-a.e. infinitesimal Euclideanity and the ssp imply the existence of non-trivial isometries with arbitrarily big measure of their fixed point set. Moreover, we can verify that isometries with this property generate small subgroups. This is shown in Propositions 3.1 and 3.6 which contain most of the work needed for the proof of Theorem 1.1. Subsequently we show that the existence of a single non-trivial isometry with a fixed point set of positive measure implicates that optimal plans are not unique, thus we can conclude Theorem 1.4.

The use of blow-up arguments is common in the proofs done for Alexandrov and Ricci limit spaces. However the delicate point is to guarantee, relying simply on the tools at hand, a non-trivial convergence of subgroups of isometries acting on sequences of scaled spaces. For Alexandrov spaces one uses the fact that geodesics do not branch, whereas in the case of Ricci limit spaces, a crucial step depends on the connectedness properties of the regular set. There exist examples in the setting of Theorem 1.1 where these properties simply do not hold. Therefore we must give new arguments; we make use of optimal transport tools and measure properties of the regular set.

During the completion of this manuscript Guijarro and Santos-Rodríguez proved in [17, Theorem 1] that $\mathrm{ISO}_{\mathfrak{m}}(X)$ is a Lie group for RCD*-spaces, compare with Corollary 1.2 above. The proof of Theorem 1 of [17] and that of our Theorem 1.1 both rely on the aforementioned result due to Gleason and Yamabe, however the approaches to the problem are different. Guijarro and Santos-Rodríguez adapted the approach of [12] in which the existence of a splitting theorem is indispensable, thus only RCD*-spaces are contemplated. In contrast, besides considering independently the groups $\operatorname{ISO}(X)$ and $\operatorname{ISO}_{\mathfrak{m}}(X),{ }^{2}$ we present a more general and direct method, which allows us to achieve the characterization stated in Theorem 1.1. Consequently, the major practical advantage is that our results are valid in a larger class of metric measure spaces: we are not restricted to work with spaces having solely Eulcidean tangents or in which a splitting theorem holds, see Remark 4.6. For example, we study spaces with weaker curvature-dimension conditions and, with more generality, spaces with good optimal transport behavior.

In the next section we give definitions and background results that will be used. In Section 3 we find Propositions 3.1 and 3.6. The rest of the work needed to conclude the proofs, and the proofs themselves, of Theorems 1.1 and 1.4, are in Section 4. At the end of the manuscript we present an example of an MCP-space where $\operatorname{ISO}_{\mathfrak{m}}(X)$ is not a Lie group.

\section{Preliminaries}

We set notation and compile definitions and results used in the paper. The text is mainly selfcontained, however, we provide references for some more elaborate definitions to maintain brevity.

\subsection{Metric Measure Spaces}

A metric measure space, $(X, \mathrm{~d}, \mathfrak{m})$, is a triple where

$(X, \mathrm{~d})$ is a complete, separable metric space and,

$\mathfrak{m} \neq 0$ is a non-negative Borel measure finite on every bounded set.

\footnotetext{
${ }^{2} \mathrm{~A}$ discussion on the relevance of considering these groups independently is found in Remark 4.7.
} 
We write mms for short. A pointed metric measure space, $(X, \mathrm{~d}, \mathfrak{m}, x)$, is a mms together with a base point $x \in X$. In the text a geodesics is a map, $\gamma:[0,1] \rightarrow X$, such that:

$$
\mathrm{d}\left(\gamma_{r}, \gamma_{s}\right)=(r-s) \mathrm{d}\left(\gamma_{0}, \gamma_{1}\right) \quad \text { for all } 0 \leq s \leq r \leq 1
$$

where $\gamma_{t}:=\gamma(t)$. We write $\operatorname{Geo}(X)$ for the space of all geodesics on $X$ endowed with topology of uniform convergence. A metric space is called a geodesic space if for every given pair of points $x, y \in(X, \mathrm{~d})$ there exists a geodesic that joins $x$ and $y$. For $t \in[0,1]$ define the evaluation map, $\mathrm{e}_{t}: \operatorname{Geo}(X) \rightarrow X$, as $\mathrm{e}_{t}(\gamma):=\gamma_{t}$ for $\gamma \in \operatorname{Geo}(X)$. The restriction map, rest $t_{s}^{t}: \operatorname{Geo}(X) \rightarrow \operatorname{Geo}(X)$, is defined as rest $t_{s}^{t}(\gamma):=\gamma \circ f_{s}^{t}$ for $s, t \in$ $[0,1], \gamma \in \operatorname{Geo}(X)$ and the real function $f_{s}^{t}(x):=(t-s) x+s$.

Two $\mathrm{mms}\left(X_{1}, \mathrm{~d}_{1}, \mathfrak{m}_{1}\right),\left(X_{2}, \mathrm{~d}_{2}, \mathfrak{m}_{2}\right)$ are isomorphic if there exists an isometry

$$
\begin{aligned}
& f: \operatorname{supp}\left(\mathfrak{m}_{1}\right) \rightarrow X_{2} \quad \text { such that } \\
& (f)_{\# \mathfrak{m}_{1}=\mathfrak{m}_{2},}
\end{aligned}
$$

where we have denoted the pushforward of a measure $v$ under a map $g$ by $(g)_{\#} v$. We use the word isometry to make reference to usual metric isometries. In contrast, we refer to maps satisfying (2.1) as measure-preserving isometries. Particularly, we note that an isometry is defined on the whole space $X_{1}$ and does not necessarily satisfy (2.1). By definition $(X, \mathrm{~d}, \mathfrak{m})$ is always isomorphic to $(\operatorname{supp}(\mathfrak{m}), \mathrm{d}, \mathfrak{m})$. This induces a canonical equivalence class of isometric metric measure spaces where only the support of the measure is relevant. We assume that $\operatorname{supp}(\mathfrak{m})=X$, which is a natural restriction in the class of isomorphisms of mms. We endow the groups $\operatorname{ISO}(X)$ and $\operatorname{ISO}_{\mathfrak{m}}(X)$ with the compactopen topology making them topological groups, see [22] pp.46. We write in the remainder $\mathrm{G} \in\left\{\operatorname{ISO}(X), \operatorname{ISO}_{\mathfrak{m}}(X)\right\}$ to denote one of these two groups.

Remark 2.2 (Topology on $\operatorname{ISO}_{\mathfrak{m}}(X)$ ) We explain and motivate our choice of topology on $\operatorname{ISO}_{\mathfrak{m}}(X)$. For locally compact metric spaces it's natural to endow $\operatorname{ISO}(X)$ with the compact-open topology since the structure under study is of pure metric nature. In addition, in this context, the rigidity of the isometries assures that pointwise convergence implies convergence w.r.t. the compact-open topology. ${ }^{3}$ Alternatively, on mms there is additional structure of interest, namely, the measure structure. However, as we explain below, the rigidity of the measure-preserving isometries guarantee that a reasonable choice of topology on $\mathrm{ISO}_{\mathfrak{m}}(X)$ coincides with the compact-open topology.

We first observe that topology that only considers the measure structure is too coarse for our purposes because it doesn't see metric properties. A logical way to proceed would be to couple a measure-wise and a metric-wise topology. However, the weakest metric convergence, the pointwise convergence, coincides with the compact-open convergence. On the other hand, in Lemma 4.5 we show that the compact-open convergence of a sequence of measure-preserving isometries, $\left(f_{n}\right)$, implies the weak convergence of the pushforward measures $\left(f_{n}\right)_{\#}(\mathfrak{m})$ in a locally compact $\mathrm{mms}$.

We will study group actions on sequences of pointed metric spaces. In this framework the pointed Gromov-Hausdorff ( $\mathrm{pGH}$ ) and pointed equivariant Gromov-Hausdorff convergence

\footnotetext{
${ }^{3}$ Rigorously we would have to justify the use of sequences to compare topologies. This can be done because $\operatorname{ISO}(X)$ is second-countable which can be concluded from the fact that $X$ is a locally compact metric space. Consult for instance [22] pp. 46.
} 
(peGH) provide canonical types of convergence. We racall rapidly these concepts and refer to $[3,10,11,18]$ for more details.

Let us first denote the set of isometry classes of compact metric measure spaces by $\mathcal{M}^{\mathrm{c}}$. We also consider triples $(X, \mathrm{~d}, \mathrm{H})$, where $(X, \mathrm{~d})$ is a compact metric space and $\mathrm{H} \leq$ $\operatorname{ISO}(X)$ is a closed subgroup and say that two triples are equivalent if they are equivariantly isomorphic up to automorphisms of the groups. We denote the equivalence classes of these triples by $\mathcal{M}_{\text {eq }}^{\mathrm{c}}$.

Definition 2.3 ( $\epsilon$-(equivariant) Gromov-Hausdorff approximation) Let $\left(X, \mathrm{~d}_{X}\right),\left(Y, \mathrm{~d}_{Y}\right) \in$ $\mathcal{M}^{\mathrm{c}}$ be metric spaces. An $\epsilon$-Gromov-Hausdorff approximation is a function $f: X \rightarrow$ $Y$ such that, for all $p, q \in X$ it holds that $\left|\mathrm{d}_{X}(p, q)-\mathrm{d}_{Y}(f(p), f(q))\right| \leq \epsilon$ and an $\epsilon$ neighborhood of $f(X)$ covers all of $\mathrm{Y}$.

Let $\left(X, \mathrm{~d}_{X}, \mathrm{H}_{X}\right),\left(Y, \mathrm{~d}_{Y}, \mathrm{H}_{G}\right) \in \mathcal{M}_{\mathrm{eq}}^{\mathrm{c}}$. An $\epsilon$-equivariant Gromov-Hausdorff approximation is a triple of functions $(f, \varphi, \psi)$ where $f: X \rightarrow Y, \varphi: \mathrm{H}_{X} \rightarrow \mathrm{H}_{Y}$ and $\psi: \mathrm{H}_{Y} \rightarrow \mathrm{H}_{X}$ such that

- $f$ is a Gromov-Hausdorff $\epsilon$-approximation;

- $\quad$ if $h_{X} \in \mathrm{H}_{X}$, and $x \in X$, then $\mathrm{d}\left(f\left(h_{X} x\right), \varphi\left(h_{X}\right) f(x)\right)<\epsilon$; and

- $\quad$ if $h_{Y} \in H_{Y}$ and $x \in X$, then $\mathrm{d}\left(f\left(\psi\left(h_{Y}\right) x\right), h_{Y} f(x)\right)<\epsilon$.

The distances are defined as follow.

Definition 2.4 ((equivariant) Gromov-Hausdorff distance) The Gromov-Hausdorff distance $\mathrm{d}_{\mathrm{GH}}$ between two compact metric spaces $\left(X, \mathrm{~d}_{X}\right)$ and $\left(Y, \mathrm{~d}_{Y}\right)$ is defined as the infimum of all $\epsilon$ 's such that there are $\epsilon$-Gromov-Hausdorff approximations from $X \rightarrow Y$ and from $Y \rightarrow X$.

The equivariant Gromov-Hausdorff distance between two triples $\left(X, \mathrm{~d}_{x}, \mathrm{H}_{X}\right)$ and $\left(Y, \mathrm{~d}_{Y}, \mathrm{H}_{Y}\right)$ is defined analogously using $\epsilon$-equivariant Gromov-Hausdorff approximations.

If $\lim _{i \rightarrow \infty} \mathrm{d}_{\mathrm{GH}}\left(X_{i}, X\right)=0$, we say that the sequence of metric spaces $\left\{\left(X_{i}, \mathrm{~d}_{X_{i}}\right)\right\}_{i \in \mathbb{N}}$ converges to the metric space $\left(X, \mathrm{~d}_{X}\right)$; equivariant Gromov-Hausdorff convergence for sequences of triples in $\mathcal{M}_{\mathrm{eq}}^{\mathrm{c}}$ is defined analogously. Furthermore, convergence for noncompact proper pointed spaces is defined by requiring the approximations to preserve the base point. In this case we say that $\left(X, \mathrm{~d}_{X}, x\right)$ converges in the pointed Gromov-Hausdorff topology to $\left(Y, \mathrm{~d}_{X}, y\right)$ if $\left(\bar{B}_{r}(x), \mathrm{d}_{X}\right) \stackrel{\mathrm{GH}}{\longrightarrow}\left(\bar{B}_{r}(y), \mathrm{d}_{Y}\right)$ for all radius $r$, under the requirement that $f(x)=y$ for all Gromov-Hausdorff $\epsilon$-approximations. In order to keep track of base points in the equivariant convergence, let us call $\mathcal{M}_{\mathrm{eq}, \mathrm{p}}^{\mathrm{c}}$ the set of equivalence classes of quadruples $(M, \mathrm{~d}, \mathrm{H}, x)$, for $(M, \mathrm{~d}, \mathrm{H}) \in \mathcal{M}_{\mathrm{eq}}^{\mathrm{c}}$ and $x \in M$, under the equivalence given by equivariant isomorphisms (up to group automorphisms) that fix the base point. The pointed eGH-convergence is then defined in terms of eGH $\epsilon$-approximations that fix base points.

Lastly, in the framework of metric measure spaces the ad hoc convergence is given by the pointed measured Gromov-Hausdorff convergence. This convergence requires pGHconvergence of the underlying metric spaces and additionally weak convergence of the pushforward measures under the $\epsilon_{n}$-Gromov-Hausdorf approximations to the reference measure of the limit space. An comprehensive reference for this topic is [15].

Any pGH-limit of a sequence of scaled spaces, $\left(X, \frac{1}{r_{i}} \mathrm{~d}, x\right) \stackrel{\mathrm{pGH}}{\rightarrow}\left(X_{x}, \mathrm{~d}_{\infty}, x_{\infty}\right)$ for $r_{i} \rightarrow$ 0 , is called a (metric) tangent cone of $X$ at $x$. We denote the set of all tangent cones of $X$ at $x$ by $\operatorname{Tan}(X, x):=\left\{\left(X_{\infty}, \mathrm{d}_{\infty}, x_{\infty}\right)\right.$ is a $\mathrm{pGH}$-limit as above $\}$. The existence or uniqueness 
of tangent cones is not guaranteed in a general setting. However, the set of points of $X$ with unique Euclidean tangent cones is called the regular set of $X$, written as $\mathcal{R}$. In detail,

$$
\mathcal{R}:=\left\{x \in X \| \exists k=k(x) \in \mathbb{N} \text { such that } \operatorname{Tan}(X, x)=\left\{\left(\mathbb{R}^{k}, d_{E}, \overline{0}\right)\right\}\right\} .
$$

We say that $(X, \mathrm{~d}, \mathfrak{m})$ has $\mathfrak{m}$-a.e. Euclidean tangents if the set of numbers $\{k(x) \in$ $\left.\mathbb{N} \| x \in \mathcal{R}, \operatorname{Tan}(X, x)=\left\{\left(\mathbb{R}^{k(x)}, d_{E}, \overline{0}\right)\right\}\right\}$ is finite and if $\mathfrak{m}(X \backslash \mathcal{R})=0$. For a fixed $\epsilon>0$, the $\epsilon$-regular set, $\mathcal{R}_{\epsilon}$, is the set $\mathcal{R}_{\epsilon}:=\cup_{\delta}(\mathcal{R})_{\epsilon, \delta}$, where for a given $\delta>0$ the set $(\mathcal{R})_{\epsilon, \delta}$ is defined as all points $x \in X$ for which there exists a $k=k(x)$ such that

$$
\mathrm{d}_{\mathrm{GH}}\left(B_{r}(x), B_{r}^{k}(\overline{0})\right)<\epsilon r \quad \text { for all } r<\delta .
$$

Above $\mathrm{d}_{\mathrm{GH}}$ is the Gromov-Hausdorff distance and $B_{r}^{k}(\overline{0}) \subset \mathbb{R}^{k}$ is the ball of radius $r$ around $\overline{0} \in \mathbb{R}^{k}$. Note that $\mathcal{R}=\cap_{\epsilon} \mathcal{R}_{\epsilon}$ and that for every $\epsilon>0$ the measure $\mathfrak{m}\left(X \backslash \mathcal{R}_{\epsilon}\right)=0$ if $X$ has $\mathfrak{m}$-a.e. Euclidean tangents.

\subsection{Lie Groups}

Denote by $G_{0}$ the identity component of $G$, that is, the largest connected set containing the identity element $\mathbb{I}$. As definition we say that $G$ is a Lie group if and only if $G / G_{0}$ is discrete $^{4}$ and the identity component $G_{0}$ is a Lie group in the usual smooth sense. We know by Remark 2.8 that $G$ looks, in the worst cases, as countable copies of a smooth Lie group which do not accumulate.

Theorem 2.5 (van Dantzig and van der Waerden [8]) Let $(X, d)$ be a connected, locally compact metric space. Then $\mathrm{ISO}(X)$ is locally compact with respect to the compact-open topology. Furthermore if $X$ is compact, then $\operatorname{ISO}(X)$ is compact.

A topological group $G$ has the no small subgroup property if there exists a neighborhood of the identity with no non-trivial subgroup. In this case we write nssp for short. Below we cite an outstanding result that characterizes Lie groups in terms of the nssp.

Theorem 2.6 (Gleason [13], Yamabe [35]) Let G be a locally compact, topological group. Then $G$ is a Lie group if and only if it has the no small subgroups property.

Remark 2.7 In [35] Yamabe generalizes Gleason's theorem to the infinite dimensional case, however, $G$ is assumed to be connected. We present an argument, due to an undisclosed Russian mathematician, which shows that we can consider non-connected groups.

An equivalent way of stating Theorem 2.6 is: Assuming the same hypothesis, then there exists an open subgroup $G^{\prime}<G$ such that for every neighborhood of the identity $U \subset G$ there exists a normal subgroup ( $U \supset) K \unlhd G$ that makes $G^{\prime} / K$ a Lie Group [34]. If $G$ has the nssp the only small normal subgroup is $\mathbb{I}$ itself, thus making $G^{\prime}$ a Lie group which by definition means that $G^{\prime} / G_{0}$ is discrete. However, this implies that $G / G_{0}$ is discrete since $G / G^{\prime}$ is also discrete and $G / G^{\prime}=\left(G / G_{0}\right) /\left(G^{\prime} / G_{0}\right)$.

Remark 2.8 We make another observation regarding the cardinality of $G / G_{0}$. In principle the group of components might be uncountable but fortunately, we can also discard this

${ }^{4}$ For a comment on the cardinality of $G / G_{0}$ see Remark 2.8 . 
behavior. Assume that $G$ is a second-countable Lie group. By definition $G / G_{0}$ is discrete which is equivalent to $G_{0}$ being open. In turn, this implies that the quotient map is open and it follows that $G / G_{0}$ is second-countable since by assumption $G$ is second-countable. A second-countable space is separable, and discrete separable spaces are countable. Finally, we recall that $\operatorname{ISO}(X)$ is second-countable for a locally compact, connected metric space.

\subsection{Curvature Dimension Conditions}

Curvature-dimension conditions require certain convexity behavior of an entropy functional defined on the space of probability measures of an mms. Different choices of entropy functional and different types of convexity conditions give rise to alternative versions of curvature-dimension conditions. However, all conditions are compatible with lower Ricci bounds in the smooth framework and are stable under pointed measured-Gromov-Hausdorff convergence. Optimal transport theory provides an appropriate framework to define these type of conditions.

We've decided not to include the definitions of the RCD*- or the $C D^{*}$-conditions since we don't use them explicitly and are rather technical. Alternatively, we present the results that show that these spaces satisfy the assumptions of Theorem 1.4. Nevertheless, let's do recall that the RCD*-condition condition was introduced in [2] and further developed in [1] and [9] to which the reader is referred to for a comprehensive discussion on the subject. The RCD*-condition itself couples a curvature-dimension condition with an infinitesimal Riemannian behavior known as infinitesimally Hilbertianity.

Let $\mathcal{P}(X)$ be the space of probability measures on $(X, \mathrm{~d})$ and $\mathcal{P}_{2}(X) \subset \mathcal{P}(X)$ the subspace of measures with finite second moments. For $\mu_{0}, \mu_{1} \in \mathcal{P}_{2}(X)$ the Wasserstein squared distance is defined as

$$
W_{2}^{2}\left(\mu_{0}, \mu_{1}\right):=\inf _{\sigma} \int_{X \times X} \mathrm{~d}(x, y)^{2} \mathrm{~d} \sigma(x, y) .
$$

The infimum taken over all measures $\sigma \in \mathcal{P}(X \times X)$ with first and second marginals equal to $\mu_{0}$ and $\mu_{1}$ respectively. If there exists a measurable function $G: X \rightarrow X$ such that the measure $\sigma=(\mathbb{I}, G)_{\#} \mu_{0}$ is a minimum we call $\sigma$ an optimal map. Given $\mu_{0}, \mu_{1} \in \mathcal{P}_{2}(X)$ the set $\operatorname{OptGeo}\left(\mu_{0}, \mu_{1}\right) \subset \mathcal{P}(\operatorname{Geo}(X))$ is defined as the set of all measures $\pi$ such that the pushforward $\left(\mathrm{e}_{0}, \mathrm{e}_{1}\right)_{\# \pi} \in \mathcal{P}(X \times X)$ realizes the minimum in Eq. 2.9. A measure $\pi \in \operatorname{OptGeo}\left(\mu_{0}, \mu_{1}\right)$ is called an optimal geodesic plan and if such a measure $\pi$ is the lift of an optimal map we call it an optimal geodesic map. Note that the existence of optimal maps is rare however, the next theorem shows their existence in mms that satisfy a curvature-dimension condition and that do not branch too much. Recall that the essentially non-branching condition says that geodesics do not branch too often, we refer to the cited articles for a precise definition.

Theorem 2.10 (Existence of optimal maps. Cavalleti-Gigli-Mondino-Rajala-Sturm [5, 16]) Let $K \in \mathbb{R}, N \in[1, \infty)$ and $(X, \mathrm{~d}, \mathfrak{m})$ be an essentially non-branching $\operatorname{MCP}(K, N)$ space. Then, for every $\mu_{0}(\ll \mathfrak{m}), \mu_{1} \in \mathcal{P}_{2}(X)$, there exist a unique optimal geodesic plan $\pi \in$ $\operatorname{OptGeo}\left(\mu_{0}, \mu_{1}\right)$. Furthermore, such $\pi$ is given by a map. In particular, there exists $\Gamma \subset$ $\operatorname{Geo}(X)$ with $\pi(\Gamma)=1$ such that the map $e_{t}: \Gamma \rightarrow X$ is injective for all $t \in[0,1)$.

We recall that essentially non-branching MCP space include: RCD*-spaces, essentially non-branching $C D^{*}$-spaces, and essentially non-branching $C D$-spaces. 
The existence of ma.e. Euclidean tangents in RCD*-spaces was proved in [14], and later the uniqueness was shown by Mondino and Naber as a byproduct of the rectifiability of $\operatorname{RCD}^{*}(K, N)$-spaces as metric spaces (compare with [21] for rectifiability as mms). A weaker version of their result is enough for us.

Theorem 2.11 ( $\mathfrak{m}$-a.e. Euclidean tangents in $\operatorname{RCD}^{*}(K, N)$-spaces. Mondino-Naber [25]) Let $K, N \in \mathbb{R}, N \geq 1$ and $(X, \mathrm{~d}, \mathfrak{m})$ be an $\operatorname{RCD}^{*}(K, N)$ space. Then $X$ has $\mathfrak{m}$-a.e. Euclidean (metric) tangents, i.e. $\mathfrak{m}(X \backslash \mathcal{R})=0$.

In the last section we will work with Ohta's definition of the $\operatorname{MCP}(K, N)$-condition. Intuitively, it requires contraction properties of measures as the $R C D^{*}$-condition but only for starting $\delta$ measures. The condition is stable w.r.t. to pmGH-convergence [27]. We give the specific shape of the condition for the case we will study.

Definition 2.12 $(\mathrm{MCP}(2,3)$-condition) A mms, $(X, \mathrm{~d}, \mathfrak{m})$, has the (2,3)-measure contraction property, $\operatorname{MCP}(2,3)$, if for every point $x \in X$ and a measurable set $A \subset X$ with $0<\mathfrak{m}(A)<\infty$ and $A \subset B_{\pi}(x)$ there exists a probability measure $\pi \in \mathcal{P}(\operatorname{Geo}(X))$ such that $\left(e_{0}\right)_{\#} \boldsymbol{\pi}=\delta_{x},\left(e_{1}\right)_{\#} \boldsymbol{\pi}=\left.\mathfrak{m}(A)^{-1} \mathfrak{m}\right|_{A}$, and

$$
\left(e_{t}\right)_{\#}\left(t \frac{\sin ^{2}(t l(\gamma))}{\sin ^{2}(l(\gamma))} \mathfrak{m}(A) \mathrm{d} \boldsymbol{\pi}(\gamma)\right) \leq \mathrm{dm} \quad t \in[0,1] .
$$

\section{Metric Measure Spaces with G Containing Small Subgroups}

We study mms where $\mathrm{G}$ has small subgroups. Granted that $\mathrm{G}$ has the small subgroup property we show the existence of many automorphisms with a large fixed point set, and vice versa: these type of automorphisms create small subgroups. After having shown this connection, the proof of Theorem 1.1 will follow without much additional work, which we present in the next section.

Define for $r>0, x \in X$, and a subgroup $\Lambda \leq \S \operatorname{ISO}(X)$ :

$$
D_{\Lambda}(r, x):=\sup _{g \in \Lambda} \sup _{y \in B \frac{r}{2}(x)} \mathrm{d}(y, g(y)) .
$$

For fixed $\Lambda$, the function $D_{\Lambda}(r, x)$ is continuous in $r$ and $x$ as long as every closed ball in $X$ is the closure of its interior [4]. This holds true when $X$ is a length space, for example. We do not assume a fully supported measure in the coming proposition.

Proposition 3.1 Let $(X, \mathrm{~d}, \mathfrak{m})$ be a $\mathrm{mms}$ where every closed ball coincides with the closure of the open ball. Assume that $X$ has $\mathfrak{m}$-a.e. Euclidean tangents. If $\mathrm{G}$ has the small subgroups property, then for every $x \in X, 0<s$, and $0<\xi<1$ there exists a non-trivial subgroup $\Lambda=\Lambda_{x, s, \xi} \subset \mathrm{G}$ such that for every $g \in \Lambda$

$$
\mathfrak{m}\left(\operatorname{Fix}(g) \cap B_{s}(x)\right) \geq \xi \mathfrak{m}\left(B_{s}(x)\right) .
$$

Proof We assume that $\mathfrak{m}\left(B_{S}(x)\right)>0$ since the inequality above trivially holds true otherwise. We argue by contradiction. The strategy is the following: assuming that inequality 
(3.2) doesn't hold we will find for every $\epsilon>0$ a quadruple $\left(\delta_{\epsilon}, r_{\epsilon}, x_{\epsilon}, \Lambda_{\epsilon}\right) \in\left(\mathbb{R}^{+}\right)^{2} \times X \times 2^{G}$ with the following properties:

- $0<r_{\epsilon} \leq \delta_{\epsilon}$

- $x_{\epsilon} \in(\mathcal{R})_{\epsilon, \delta_{\epsilon}}$

- $\Lambda_{\epsilon} \leq \operatorname{ISO}(X)$ is a subgroup

- $D_{\Lambda_{\epsilon}}\left(r_{\epsilon}, x_{\epsilon}\right)=\frac{r_{\epsilon}}{20}$.

The existence of such a family of quadruples would lead to a contradiction and thus, would prove the proposition. Indeed, observe that if for every $\epsilon>0$ there exists a quadruple as above, then for a sequence $\epsilon_{n} \rightarrow 0$ there exists a subsequence $\epsilon_{n}$ (denoted in the same way) such that, in the eGH-sense, the scaled spaces below converge to

$$
\left(B_{r_{\epsilon}}\left(x_{\epsilon}\right), \frac{1}{r_{\epsilon}} \mathrm{d}, \Lambda_{\epsilon}\right) \stackrel{\mathrm{eGH}}{\longrightarrow}\left(B_{1}(\overline{0}) \subset \mathbb{R}^{k}, \mathrm{~d}_{E}, \Lambda_{\infty}\right),
$$

where $k \in \mathbb{N}$, and $\Lambda_{\infty} \leq \operatorname{ISO}\left(\mathbb{R}^{k}\right)$ is a non-trivial subgroup satisfying $D_{\Lambda_{\infty}}(1, \overline{0})=\frac{1}{20} .5$ This creates the contradiction, since every non-trivial subgroup of Euclidean isometries $\mathrm{H}$ fulfills $D_{\mathrm{H}}(1, \overline{0})>\frac{1}{20}{ }^{6}$

We proceed to construct a family of quadruples satisfying conditions (3.3). Suppose that (3.2) does not hold. That is, there exist $x \in X, 0<s$, and $0<\xi<1$ such that for every non-trivial subgroup $\mathrm{H} \subset \mathrm{G}$ there exists an $f \in \mathrm{H}$ where

$$
\mathfrak{m}\left(\operatorname{Fix}(f) \cap B_{S}(x)\right)<\xi \mathfrak{m}\left(B_{S}(x)\right) .
$$

Note that necessarily $f \neq \mathbb{I}$. Take $\epsilon>0$ and choose small enough $\delta_{\epsilon} \in \mathbb{R}$ so that $0<\delta_{\epsilon}<s$, and

$$
\xi \mathfrak{m}\left(B_{S}(x)\right)<\mathfrak{m}\left((\mathcal{R})_{\epsilon, \delta_{\epsilon}} \cap B_{S}(x)\right) .
$$

The $\mathfrak{m}$-a.e. Euclidean tangents of $X$, together with the continuity from below of the measure and the fact that $\mathcal{R} \subset(\mathcal{R})_{\epsilon}=\cup_{\delta>0}(\mathcal{R})_{\epsilon, \delta}$ make possible the choice of such a $\delta_{\epsilon}$. Indeed, since for $\delta^{\prime} \leq \delta^{\prime \prime}$ it holds that $(\mathcal{R})_{\epsilon, \delta^{\prime \prime}} \subset(\mathcal{R})_{\epsilon, \delta^{\prime}}$ we can write $(\mathcal{R})_{\epsilon}=\cup_{n \in \mathbb{N}}(\mathcal{R})_{\epsilon, 1 / n}$ as a countable union of sets. Now just notice that

$$
\begin{aligned}
\mathfrak{m}\left(B_{s}(x)\right) & =\mathfrak{m}\left(B_{s}(x) \cap \mathcal{R}_{\epsilon}\right)=\mathfrak{m}\left(B_{S}(x) \cap\left(\cup_{n \in \mathbb{N}}(\mathcal{R})_{\epsilon, 1 / n}\right)\right) \\
& =\lim _{n \rightarrow \infty} \mathfrak{m}\left(\cup_{j \leq n}\left(B_{S}(x) \cap(\mathcal{R})_{\epsilon, 1 / j}\right)\right) \\
& =\lim _{n \rightarrow \infty} \mathfrak{m}\left(B_{S}(x) \cap(\mathcal{R})_{\epsilon, 1 / n}\right) .
\end{aligned}
$$

Choose $n \in \mathbb{N}$ big enough and take $\delta_{\epsilon}<\min \{s, 1 / n\}$.

\footnotetext{
${ }^{5}$ In detail, the definition of the sets $(\mathcal{R})_{\epsilon, \delta_{\epsilon}}$ and assumption about $X$ having $\mathfrak{m}$-a.e. Euclidean tangents guarantee the existence of a subsequence $\epsilon_{n}^{\prime}$ for which $\left(B_{r_{\epsilon_{n}^{\prime}}}\left(x_{\epsilon}\right), \frac{1}{r_{\epsilon_{n}^{\prime}}} \mathrm{d}\right) \stackrel{\mathrm{GH}}{\longrightarrow}\left(B_{1}(\overline{0}) \subset \mathbb{R}^{k}, \mathrm{~d}_{E}\right)$ for some $k \in \mathbb{N}$; thus, the claim is concluded using [11, Proposition 3.6]. The continuity of $\mathrm{D}: \mathcal{M}_{\text {eq.p }}^{\mathrm{c}} \rightarrow \mathbb{R}$ : $\left(X_{n}, \mathrm{~d}_{n}, \Lambda_{n}, x_{n}\right) \mapsto D_{\Lambda_{n}}\left(1, x_{n}\right)$ under (pointed) eGH-convergence, which follows from the definitions, implies that $D_{\Lambda_{\infty}}(1, \overline{0})=1 / 20$. Details on equivariant GH-convergence are found in Section 2 .

${ }^{6}$ The existence of a lower bound for $\mathrm{D}_{\mathrm{H}}(1, \overline{0})$ is a consequence of the nssp of the Lie group $\operatorname{ISO}\left(\mathbb{R}^{N}\right)$; the number $1 / 20$ simply serves as a bound. To corroborate this fact it's sufficient to consider subgroups which are generated by a non-trivial isometry $g \in \operatorname{ISO}\left(\mathbb{R}^{N}\right)$. Since $g$ is a composition of translations and elements of $\mathrm{O}(N)$, and subgroups of translations have unbounded displacement, it's enough to show the bound for $g \in \mathrm{O}(N)$. After recalling that (for some orthonormal frame) $g$ has the matrix representation $\operatorname{DIAG}\left(\alpha_{1}, \cdots, \alpha_{k}, \pm 1, \cdots, \pm 1\right)$, where the $\alpha_{i}$ 's are $2 \times 2$ rotation matrices, the claim is easily verified by restricting the analysis to rotations in the plane and reflections along $(N-1)$-hyperplanes.
} 
Inequality (3.4) combined with the reductio ad absurdum assumption imply that for every non-trivial $\mathrm{H} \leq \mathrm{ISO}(X)$ there exist $f(\neq \mathbb{I}) \in \mathrm{H}$ such that the set $B_{S}(x) \cap(\mathcal{R})_{\epsilon, \delta_{\epsilon}} \backslash$ $\operatorname{Fix}(f)$ is not empty.

In view of the small subgroups property of $\mathrm{G}$, we can find a non-trivial small subgroup

$$
\begin{aligned}
\Lambda_{\epsilon} \subset U_{\epsilon} & :=\left\{g \in \mathrm{G} \| \sup _{y \in B_{2 s}(x)} \mathrm{d}(y, g(y))<\frac{\delta_{\epsilon}}{20}\right\} \\
& =\left\{g \in \mathrm{G} \| g(y) \in B_{\delta_{\epsilon} / 20}(y) \text { for all } y \in \overline{B_{2 s}}(x)\right\} .
\end{aligned}
$$

In particular, there exist $g(\neq \mathbb{I}) \in \Lambda_{\epsilon}$ and $x_{\epsilon} \in B_{S}(x)$ such that

$$
\begin{aligned}
x_{\epsilon} & \in B_{S}(x) \cap(\mathcal{R})_{\epsilon, \delta_{\epsilon}} \backslash \operatorname{Fix}(g) \text { and } \\
0 & <\mathrm{d}\left(x_{\epsilon}, g\left(x_{\epsilon}\right)\right)<\frac{\delta_{\epsilon}}{20} .
\end{aligned}
$$

Denote by $\theta=\theta\left(x_{\epsilon}\right):=20 \mathrm{~d}\left(x_{\epsilon}, g\left(x_{\epsilon}\right)\right)<\delta_{\epsilon}$. By construction it follows that

$$
\begin{aligned}
\frac{1}{20} \theta & \leq D_{\Lambda_{\epsilon}}\left(\theta, x_{\epsilon}\right) \\
D_{\Lambda_{\epsilon}}\left(\delta_{\epsilon}, x_{\epsilon}\right) & \leq D_{\Lambda_{\epsilon}}(4 s, x)<\frac{1}{20} \delta_{\epsilon} .
\end{aligned}
$$

Finally, the continuity of $D_{\Lambda_{\epsilon}}\left(\circ, x_{\epsilon}\right)$ and the intermediate value theorem imply that there exists $r_{\epsilon} \in \mathbb{R}$ such that $D_{\Lambda_{\epsilon}}\left(r_{\epsilon}, x_{\epsilon}\right)=\frac{1}{20} r_{\epsilon}$ for some $\theta \leq r_{\epsilon}<\delta_{\epsilon}$. Hence for $\epsilon>0$ there exists a quadruple $\left(\delta_{\epsilon}, r_{\epsilon}, x_{\epsilon}, \Lambda_{\epsilon}\right)$ satisfying (3.3).

Remark 3.5 Note that the logical negation to the conclusion of Proposition 3.1 is equivalent to condition (a) of Theorem 1.1.

Next, we see that we can generate small subgroups from the existence of automorphisms with large fixed point sets.

Proposition 3.6 Let $(X, \mathrm{~d}, \mathfrak{m})$ be a locally compact $\mathrm{mms}$ where every closed ball coincides with the closure of its interior. Then $\mathrm{G}$ has the small subgroups property if for every $x \in X$, $0<s$, and $0<\xi^{\prime}<1$ there exists a non-trivial subgroup $\Lambda=\Lambda_{x, s, \xi^{\prime}} \subset \mathrm{G}$ such that for every $g \in \Lambda$

$$
\mathfrak{m}\left(X \backslash \operatorname{Fix}(g) \cap B_{S}(x)\right) \leq \xi^{\prime} \mathfrak{m}\left(B_{S}(x)\right) .
$$

Proof We give a sequence $\left\{\xi_{N}\right\}_{N \in \mathbb{N}} \subset(0,1)$ which generates, according to the hypothesis, a sequence of non-trivial subgroups $\left\{\Lambda_{x, N, \xi_{N}}\right\}_{N \in \mathbb{N}} \leq \mathrm{G}$ such that $\Lambda_{N} \subset U_{N}(\ni \mathbb{I})$ for every $N \in \mathbb{N}$, where $\left\{U_{N}\right\}_{N \in \mathbb{N}} \subset \mathrm{G}$ is local basis of the compact-open topology at $\mathbb{I}$. Thus proving the existence of small subgroups of $\mathrm{G}$.

Accordingly we fix $x \in X, N \in \mathbb{N}$, and define

$$
\xi_{N}^{\prime}:=\mathfrak{m}\left(B_{N}(x)\right)^{-1} \inf _{y \in B_{N}(x)}\left\{\mathfrak{m}\left(B_{1 / N}(y) \cap B_{N}(x)\right)\right\} .
$$

We claim that $0<\xi_{N}^{\prime}$. Indeed, choose a converging sequence ${ }^{7} y_{m} \rightarrow y_{\infty} \in \bar{B}_{N}(x)$ such that $\left.\liminf _{m \rightarrow \infty} \mathfrak{m}\left(B_{N}(x)\right)^{-1} \mathfrak{m}\left(B_{1 / N}\left(y_{m}\right) \cap B_{N}(x)\right)\right)=\xi_{N}^{\prime} \cdot{ }^{8}$ Since the measure $\mathfrak{m}$

\footnotetext{
${ }^{7}$ Using a subsequence if necessary.

${ }^{8}$ The existence of such subsequence is guarantee since locally compact, complete metric spaces for which the closure of open balls coincides with closed balls are proper.
} 
has full support there exists a small ball, $B_{\tau}\left(y_{\infty}\right)$, with $\mathfrak{m}\left(B_{\tau}\left(y_{\infty}\right) \cap B_{N}(x)\right)>0$ which is a lower bound of $\mathfrak{m}\left(B_{1 / N}\left(y_{m}\right) \cap B_{N}(x)\right)$ for large enough $m$, hence, validating the claim. We take $0<\xi_{N}<\xi_{N}^{\prime}$ and write $\Lambda_{N}:=\Lambda_{x, N, \xi_{N}}$ for the non-trivial subgroup given by the hypothesis for the triple $\left(x, N, \xi_{N}\right)$. By construction we verify that

$$
\mathfrak{m}\left(X \backslash \operatorname{Fix}(f) \cap B_{N}(x)\right)<\mathfrak{m}\left(B_{1 / N}(y) \cap B_{N}(x)\right)
$$

for every $y \in B_{N}(x)$ and $f \in \Lambda_{N}$.

Observe now that if $\mathrm{d}(z, g(z))>2 t$ then $B_{t}(z) \cap B_{R}(x) \subset X \backslash F i x(g) \cap B_{R}(x)$ for $g \in \mathrm{G}, z \in X$, and numbers $t, R \in \mathbb{R}^{+}$. Therefore, we conclude from (3.7) that for every $y \in B_{N}(x)$ and $f \in \Lambda_{N}$ we have that $d(y, f(y)) \leq 2 / N$. Hence $\Lambda_{N}$ is contained in the neighborhood of the identity:

$$
U_{N}:=\left\{g \in \mathrm{G} \| \mathrm{d}(y, g(y))<3 / N \text { for every } y \in \bar{B}_{N}(x)\right\} .
$$

Accordingly, the proof is complete considering that the choice of $N$ was arbitrary.

\section{Proof of Theorem 1.1}

We start this section with a lemma that shows that the uniqueness of optimal geodesic maps is sufficient to guarantee that non-trivial isometries have fixed point sets of measure zero. In particular, Theorem 1.4 is concluded from this result and Theorem 1.1 after taking into consideration that locally compact, complete length spaces are geodesic.

Lemma 4.1 (Zero measure of the fixed point set) Let $(X, \mathrm{~d}, \mathfrak{m})$ be a mms such that for every $\mu_{0}, \mu_{1} \in \mathcal{P}_{2}(X)$ with $\mu_{0} \ll \mathfrak{m}$ there exists a unique optimal geodesic plan $\pi \in$ $\operatorname{OptGeo}\left(\mu_{0}, \mu_{1}\right)$. Furthermore, assume that $\pi$ is concentrated on a set of geodesics, $\Gamma \subset$ $\operatorname{Geo}(X)$, such that the map $\mathrm{e}_{0}: \Gamma \rightarrow X$ is injective. Let $f \neq \mathbb{I}$ be an isometry of $X$. Then $\mathfrak{m}(\operatorname{Fix}(f))=0$.

Proof We proceed by contradiction. Suppose that there exist $\mathbb{I} \neq f \in \operatorname{ISO}(X)$, and a set $A \subset \operatorname{Fix}(f)$ with positive measure. Let $x \in X \backslash \operatorname{Fix}(f)$ and define the probability measures $\mu_{0}:=\left.\mathfrak{m}(A)^{-1} \mathfrak{m}\right|_{A}$ and $\mu_{1}:=\frac{1}{2}\left(\delta_{x}+\delta_{f(x)}\right)$. We denote by $\pi \in \operatorname{OptGeo}\left(\mu_{0}, \mu_{1}\right)$ the unique geodesic plan between $\mu_{0}$ and $\mu_{1}$. Let $\Gamma \subset \operatorname{Geo}(X)$ be the set where $\pi$ is concentrated and where $e_{0}$ is injective. Set:

$$
\begin{array}{ll}
\Gamma_{1}:=\{\gamma \in \Gamma \quad \mid & \left.\mathrm{e}_{1}(\gamma)=x\right\} \\
\Gamma_{2}:=\{\gamma \in \Gamma \quad \mid & \left.\mathrm{e}_{1}(\gamma)=f(x)\right\} \\
A_{i}:=\mathrm{e}_{0}\left(\Gamma_{i}\right) \quad i=1,2 .
\end{array}
$$

$\Gamma_{1}\left(\Gamma_{2}\right)$ is the subset of geodesics of $\Gamma$ that end in $x \quad(f(x))$ and $A_{i}$ is the projection of $\Gamma_{i}$ onto the set $A$. We have that none of these sets are empty, that the measures $\pi\left(\Gamma \backslash\left(\Gamma_{1} \cup \Gamma_{2}\right)\right)=0=\mathfrak{m}\left(A \backslash\left(A_{1} \cup A_{2}\right)\right)$ and that $A_{1} \cap A_{2}=\emptyset$. The last fact is a consequence of the injectivity of $e_{0}$. We define now the measure $\pi^{\prime} \in \mathcal{P}(\operatorname{Geo}(X))$ as

$$
\pi^{\prime}:=\left.(\hat{f})_{\#} \pi\right|_{\Gamma_{1}}+\left.\left(\widehat{f^{-1}}\right)_{\#} \pi\right|_{\Gamma_{2}},
$$

where the bijection of $\operatorname{Geo}(X), \gamma \mapsto g \circ \gamma$, induced by some $g \in \operatorname{ISO}(X)$ is written as $\hat{g}: \operatorname{Geo}(X) \rightarrow \operatorname{Geo}(X)$. The measure $\pi^{\prime}$ is a symmetric analog of $\pi$ but $\pi^{\prime} \neq \pi$. Indeed, note that $\pi^{\prime}\left(\hat{f}\left(\Gamma_{1}\right)\right)=1 / 2 \neq 0=\pi\left(\hat{f}\left(\Gamma_{1}\right)\right)$ because by construction $\hat{f}\left(\Gamma_{1}\right) \cap \Gamma_{i}=\emptyset$, for $i=1,2$. To verify the latter claim it is sufficient to observe that geodesics contained in 
$\hat{f}\left(\Gamma_{1}\right)$ have distinct starting points than geodesics contained in $\Gamma_{2}$ and moreover, different terminal points than geodesics in $\Gamma_{1}$.

We claim that $\pi^{\prime} \in \operatorname{OptGeo}\left(\mu_{0}, \mu_{1}\right)$ is also a dynamical plan. This would contradict the hypothesis of the uniqueness of $\pi$ and finish the proof of the lemma. We proceed to verify the claim.

We need to show that $\pi^{\prime}$ minimizes $\int_{\operatorname{Geo}(X)} l^{2}(\gamma) d \rho$, where we have written $l^{2}(\gamma)$ for the squared length of $\gamma$. The minimum taken over all measures $\rho \in \mathcal{P}(\operatorname{Geo}(X))$ such that $\left(e_{i}\right)_{\#} \rho=\mu_{i}$ for $i=0,1$. We check that the pushforwards of $\pi^{\prime}$ under the evaluation map are as above. For this we observe that for $g \in \operatorname{ISO}(X), B \subset X$, and $t \in[0,1]$

$$
\begin{aligned}
\hat{g} \circ \mathrm{e}_{t}^{-1}(B) & =\hat{g}\left(\left\{\gamma \in \operatorname{Geo}(X) \mid \mathrm{e}_{t}(\gamma) \in B\right\}\right) \\
& =\left\{\gamma \in \operatorname{Geo}(X) \mid \mathrm{e}_{t}(\gamma) \in g(B)\right\}=\mathrm{e}_{t}^{-1} \circ g(B),
\end{aligned}
$$

and that $\hat{g}^{-1}=\widehat{g^{-1}}$. Next we compute the pushforward of $\pi^{\prime}$ under $\mathrm{e}_{t}$ :

$$
\begin{aligned}
\left(\mathrm{e}_{t}\right)_{\#} \pi^{\prime} & =\left.\left(\mathrm{e}_{t} \circ \hat{f}\right)_{\# \pi}\right|_{\Gamma_{1}}+\left.\left(\mathrm{e}_{t} \circ \widehat{f^{-1}}\right)_{\# \pi}\right|_{\Gamma_{2}} \\
& =\left.(f)_{\#}\left(\mathrm{e}_{t}\right)_{\#} \pi\right|_{\Gamma_{1}}+\left.\left(f^{-1}\right)_{\#}\left(\mathrm{e}_{t}\right)_{\#} \pi\right|_{\Gamma_{2}} .
\end{aligned}
$$

Then $\left(\mathrm{e}_{0}\right)_{\#} \pi^{\prime}=\mu_{0}$ since $\left.f\right|_{A}=\left.\mathbb{I}\right|_{A}$. As for the other pushforward we have that $\left(\mathrm{e}_{1}\right)_{\#} \pi^{\prime}=(f)_{\#}\left(\frac{1}{2} \delta_{x}\right)+\left(f^{-1}\right)_{\#}\left(\frac{1}{2} \delta_{f(x)}\right)=\frac{1}{2}\left(\delta_{x}+\delta_{f(x)}\right)=\mu_{1}$. To finish we see that $\pi^{\prime} \in \operatorname{OptGeo}\left(\mu_{0}, \mu_{1}\right)$ by showing that the value of $\int_{\mathrm{Geo}(X)} l^{2}(\gamma) \mathrm{d} \pi^{\prime}$ is the minimum of the functional.

$$
\begin{aligned}
\int_{\mathrm{Geo}(X)} l^{2}(\gamma) \mathrm{d} \pi^{\prime}(\gamma) & =\int_{\operatorname{Geo}(X)} l^{2}(\gamma) \mathrm{d}\left(\left.(\hat{f})_{\# \pi}\right|_{\Gamma_{1}}+\left.\left(\widehat{f^{-1}}\right)_{\#} \pi\right|_{\Gamma_{2}}\right)(\gamma) \\
& =\int_{\operatorname{Geo}(X)} l^{2} \circ \hat{f}(\gamma) \cdot \chi_{\Gamma_{1}}(\gamma) \mathrm{d} \pi(\gamma)+l^{2} \circ \widehat{f^{-1}}(\gamma) \cdot \chi_{\Gamma_{2}}(\gamma) \mathrm{d} \pi(\gamma) \\
& =\int_{\operatorname{Geo}(X)} l^{2}(\gamma) \cdot\left(\chi_{\Gamma_{1}}+\chi_{\Gamma_{2}}\right)(\gamma) \mathrm{d} \pi(\gamma)=\int_{\operatorname{Geo}(X)} l^{2}(\gamma) \mathrm{d} \pi(\gamma) .
\end{aligned}
$$

Remark 4.3 The hypothesis in Lemma 4.1 can be we weakened. We may require the existence of the unique geodesic plan only for final measures satisfying $\mu_{1} \ll \mathfrak{m}$ rather than for an arbitrary $\mu_{1} \in \mathcal{P}_{2}(X)$. We can repeat the proof choosing as final measure

$$
\mu_{1}:=\frac{1}{2}\left(\left.\mathfrak{m}\left(B_{r}(x)\right)^{-1} \mathfrak{m}\right|_{B_{r}(x)}+\left.\mathfrak{m}\left(f\left(B_{r}(x)\right)\right)^{-1} \mathfrak{m}\right|_{f\left(B_{r}(x)\right)}\right)
$$

where $B_{r}(x) \subset \operatorname{Fix}(f)^{c}$ is a sufficiently small ball.

Consistently with Theorem 2.10 we obtain

Corollary 4.4 Let $(X, \mathrm{~d}, \mathfrak{m})$ be an essentially non-branching $\operatorname{MCP}(K, N)$-space and $f \in$ $\operatorname{ISO}(X)$. If $\mathfrak{m}(\operatorname{Fix}(f))>0$ then $f=\mathbb{I}$.

In particular, this holds true for RCD*-spaces, essentially non-branching $\mathrm{CD}^{*}$-spaces, and essentially non-branching CD-spaces.

In order to use Gleason and Yamabe's characterization Theorem 2.6 we need to show that $G$ is a locally compact topological group. Recall that van Dantzig and van der Waerden have proved that $\operatorname{ISO}(X)$ is locally compact, granted that $X$ is locally compact and connected, see Theorem 2.5. Below we prove that $\operatorname{ISO}_{\mathfrak{m}}(X)$ is a closed subgroup of $\operatorname{ISO}(X)$. 
Lemma 4.5 Let $(X, \mathrm{~d}, \mathfrak{m})$ be a connected, locally compact $\mathrm{mms}$. Then $\operatorname{ISO}_{\mathfrak{m}}(X)$ is a locally compact closed subgroup of $\mathrm{ISO}(X)$ with respect to the compact-open topology.

Proof We show that $\operatorname{ISO}_{\mathfrak{m}}(X)$ is closed. The local compactness of $\operatorname{ISO}_{\mathfrak{m}}(X)$ follows from the fact that $\operatorname{ISO}_{\mathfrak{m}}(X)$ is a closed subgroup of a locally compact group. Let $\left(f_{n}\right)_{n \in \mathbb{N}} \subset$ $\mathrm{ISO}_{\mathfrak{m}}(X)$ be a converging sequence w.r.t. the compact-open topology with limit $f:=$ $\lim _{n \rightarrow \infty} f_{n}$. It is easy to see that $f$ is an isometry. Thus, to finish the proof, it remains to check that $(f)_{\#} \mathfrak{m}=\mathfrak{m}$. This follows from the regularity of the measure, as we argue below.

Indeed, since the measures $\left(f_{n}\right)_{\#} \mathfrak{m}=\mathfrak{m}$ are all equal, they trivially converge weakly to $\mathfrak{m}$. On the other hand we will show that the pushforward of $\mathfrak{m}$ under $f_{n}$ weakly converges to the measure $(f)_{\#} \mathfrak{m}$. Therefore, $(f)_{\#} \mathfrak{m}=\mathfrak{m}$ by the uniqueness of the limit. By using the definition of the pushforward and the continuity of $g \circ f_{n}$, it is enough to verify that for every bounded continuous function with bounded support, $g: X \rightarrow \mathbb{R}$, it holds that

$$
\lim _{n \rightarrow \infty} \int_{X} g \circ f_{n} \mathrm{dm}=\int_{X} g \circ f \mathrm{dm},
$$

to show that $\left(f_{n}\right)_{\#} \mathfrak{m} \stackrel{w}{\rightarrow}(f)_{\#} \mathfrak{m}$. After the following observation it is clear that this last equality holds.

Assuming that $g$ is as above we can construct an $m$-integrable function, $G$, such that $\left|g \circ f_{n}(x)\right| \leq G(x)$ for all $x \in X$ and make use of the dominated convergence theorem. Take for example the multiple of the characteristic function $G:=\left.k_{g} \chi\right|_{B_{r}(y)}$, where $k_{g}$ is a bound on $g$ and $r \in \mathbb{R}$ and $y \in X$ are such that $\cup_{n \in \mathbb{N}} \operatorname{supp}\left(g \circ f_{n}\right) \subset B_{r}(y)$. The existence of such a pair $\{r, y\}$ is guaranteed because $g$ has bounded support, and because $f_{n} \rightarrow f$ converges uniformly in compact subsets. The integrability of $G$ follows from $\mathfrak{m}$ being finite on bounded sets.

We have now done all the work needed to prove Theorem 1.1. Compare Theorem 1.1 to Theorem 4.5 in [4].

Proof of Theorem 1.1 Being the groups of isometries and of measure-preserving isometries locally compact spaces (Theorem 2.5, and Lemma 4.5) we can rely on Gleason and Yamabe's characterization of Lie groups. That is to say, $\mathrm{G} \in\left\{\operatorname{ISO}(X), \operatorname{ISO}_{\mathfrak{m}}(X)\right\}$ is a Lie group if and only if $G$ does not have the small subgroup property. Note that the contrapositive statements to Propositions 3.1 and 3.6 show that $\mathrm{G}$ not having the ssp is equivalent to:

(a') There exist $x \in X, 0<s, 0<\xi<1$ such that for every non-trivial subgroup $\Lambda \subset \operatorname{ISO}(X)$ there exists an isometry $g \in \Lambda$ with

$$
\mathfrak{m}\left(\operatorname{Fix}(g) \cap B_{S}(x)\right)<\xi \mathfrak{m}\left(B_{S}(x)\right) .
$$

As already observed in Remark 2.2, conditions (a) of Theorem 1.1 and ( $\left.\mathrm{a}^{\prime}\right)$ are equivalent. Indeed, it is clear that (a) implies $\left(a^{\prime}\right)$. The other implication follows after observing that the existence of an isomorphism $\mathbb{I} \neq g \in \mathrm{G}$ with $\mathfrak{m}\left(\operatorname{Fix}(g) \cap B_{S}(x)\right) \geq \xi \mathfrak{m}\left(B_{S}(x)\right)=$ Fix implies that the measure of the fix point set of every element in the generated subgroup $\langle g\rangle \neq \mathbb{I}$ is greater than or equal to Fix. This proves the first part of the theorem.

Finally, note that granted that $\operatorname{ISO}(X)$ has the no small subgroup property, then $\operatorname{ISO}_{\mathfrak{m}}(X)$ has the same property since they both are endowed with the compact-open topology. This shows that $\operatorname{ISO}_{\mathfrak{m}}(X)$ is a Lie group if $\operatorname{ISO}(X)$ is a Lie group. 
Remark 4.6 The conclusion of Theorem 1.1 remains valid under a weaker assumption on tangent cones. We say that $X$ has well-behaved tangents if it has $\mathfrak{m}$-almost everywhere unique tangent cones and the following holds:

- The set of all metric spaces that appear as unique tangent cones, $\operatorname{Tan}(\mathcal{R})$, is compact.

- There exist a constant $0<\mathrm{k}_{0}$ such that $D_{\mathrm{H}^{\infty}}\left(1, y^{\infty}\right)>\mathrm{k}_{0}$ for every non-trivial subgroup $H^{\infty} \leq \operatorname{ISO}\left(Y^{\infty}\right)$ for all $\left(Y^{\infty}, \mathrm{d}_{Y^{\infty}}, y^{\infty}\right) \in \operatorname{Tan}(\mathcal{R})$.

Indeed, if $X$ has well-behaved tangents then the only part of the argument which depends on the hypothesis on the behavior of tangent cones, the proof of Proposition 3.1, can be repeated verbatim. However, in hope of a clearer exposition we opted not to present Theorem 1.1 in full generality since the alternative proof does not contribute with new ideas. A complete exposition can be found in the author's doctoral thesis [31].

This observation becomes relevant in the study of metric measure spaces which have well-behaved tangents which might not be Euclidean. Metric measure spaces with a unique space appearing $\mathfrak{m}$-almost everywhere as tangent cone have well-behaved tangents. More examples are presented by metric measure spaces for which $\operatorname{Tan}(\mathcal{R})$ is a finite union of Euclidean spaces, normed spaces, and Carnot groups. For example, it is known that any corank 1 Carnot group of dimension $(k+1)$ equipped with a left-invariant measure is an essentially non-branching MCP-space with unique non-Euclidean tangents by work of Rizzi [29], thus their automorphism groups are Lie groups. More generally, Le Donne proved in [7] that geodesic spaces equipped with a doubling measure that have $\mathfrak{m}$-a.e. unique tangents have $\mathfrak{m}$-a.e. Carnot groups as tangents.

Remark 4.7 In general the implications

$$
\operatorname{ISO}(X) \text { is a Lie Group }(\Longleftarrow) \Longrightarrow \quad \operatorname{ISO}_{\mathfrak{m}}(X) \text { is a Lie Group. }
$$

need not hold in any direction. In Theorem 1.1 the implication to the right side can be shown relying on regularity properties of the measure. While the other direction is more drastic. There exist spaces, even with "very" regular measures, for which $\operatorname{ISO}_{\mathfrak{m}}(X)$ is a Lie group but $\operatorname{ISO}(X)$ is not.

\section{Metric Measure Spaces with $\operatorname{ISO}_{\mathfrak{m}}(X)$ Not a Lie Group}

We show that the MCP-condition is not strong enough to guarantee that the group of measure-preserving isometries is a Lie group. We also see that for a geodesic and compact mms with finite measure $\operatorname{ISO}_{\mathfrak{m}}(X)$ might fail to be a Lie group. We start by presenting a well-known example to develop intuition about the connection between $\operatorname{ISO}_{\mathfrak{m}}(X)$ not being a Lie group, $\mathrm{ISO}_{\mathfrak{m}}(X)$ having small subgroups, and the branching of geodesics.

Example 5.1 Denote the circle of radius $r$ by $S_{r}$. The Hawaiian earring, $\mathbb{H}$, is the space we obtain after gluing the circles $\left\{S_{\frac{1}{n^{2}}} \| n \in \mathbb{N}\right\}$ by identifying one point of every circle, see Fig. 1. Endow $\mathbb{H}$ with the arc-length distance $d_{\mathbb{H}}$ and the 1-dimensional Hausdorff measure $\mathcal{H}^{1}$. This makes $\left(\mathbb{H}, \mathrm{d}_{\mathbb{H}}, \mathcal{H}^{1}\right)$ a compact, geodesic metric measure space with finite measure. Observe that $\operatorname{ISO}(H)=\operatorname{ISO}_{\mathfrak{m}}(H)=\Pi^{\infty}\{ \pm 1\}$ where the compact-open topology coincides with the product topology. Hence $\operatorname{ISO}(X)$ is totally disconnected but not discrete. By definition, $\operatorname{ISO}(X)$ is not a Lie group since $\operatorname{ISO}(X) / \operatorname{ISO}(X)_{0}$ is not discrete. 


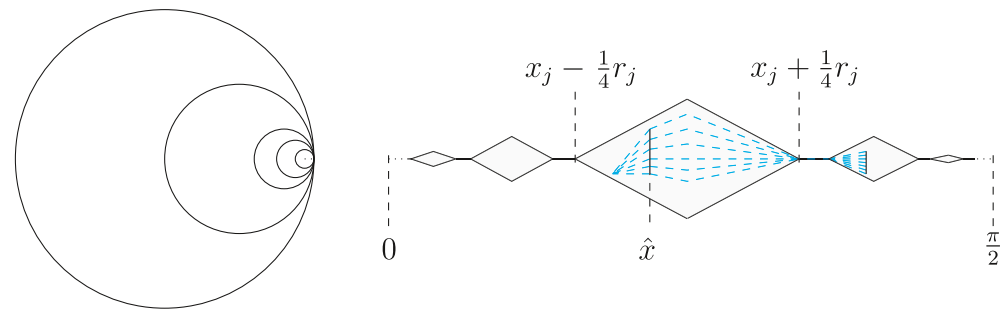

Fig. 1 Hawaiian earring $\mathbb{H}$ and fancy necklace $\mathcal{F N}$

Example 5.2 (The fancy necklace) Given $n \in \mathbb{N}$, an $n$-necklace $\left(\mathcal{N}^{n}, \mathrm{~d}_{n}, \mathfrak{m}_{n}\right) \subset \mathbb{R}^{2}$ is a mms with $n$ diamond-shaped figures, whose definition is inspired by a construction done by Ketterer and Rajala in [23]. A fancy necklace $\left(\mathcal{F} \mathcal{N}, \mathrm{d}_{\mathcal{F} \mathcal{N}}, \mathfrak{m}_{\mathcal{F N}}\right)$ is then a measured GHlimit of a sequence of $n$-necklaces $\left\{\left(\mathcal{N}^{n}, \mathrm{~d}_{n}, \mathfrak{m}_{n}\right)\right\}_{n \in \mathbb{N}}$. We begin by defining inductively the sets $\mathcal{N}^{n} \subset \mathbb{R}^{2}$ and then endowing them with a metric measure structure.

Given a sequence $\left\{\left(r_{n}, x_{n}\right)\right\}_{n \in \mathbb{N}} \subset \mathbb{R}^{2}$ (consistency conditions will be specified below), for $k \in \mathbb{N}$, we write $I_{k}=\left[x_{k}-\frac{1}{4} r_{k}, x_{k}+\frac{1}{4} r_{k}\right]$ and the diamond-shaped sets:

$$
D_{k}:=\left\{(x, y) \in \mathbb{R}^{2} \||y| \leq \frac{1}{9}\left(\frac{1}{4} r_{k}-\left|x-x_{k}\right|\right)\right\} \text {. }
$$

Set $\mathcal{N}^{0}:=[0, \pi / 2] \times\{0\} \subset \mathbb{R}^{2}$. For $n \in \mathbb{N}$, construct the $n$-necklace $\mathcal{N}^{n}$ by replacing, in the $(n-1)$-necklace $\mathcal{N}^{n-1}$, the segment $I_{n} \times\{0\} \subset \mathcal{N}^{n-1}$ with the diamond $D_{n}$. (See Fig. 1.) To have a consistent construction we require that the sequence $\left\{\left(r_{n}, x_{n}\right)\right\}_{n \in \mathbb{N}} \subset \mathbb{R}^{2}$ satisfies:

$$
\begin{aligned}
& 0<r_{n} \leq 1, \quad \frac{1}{4} r_{n} \leq x_{n} \leq \frac{\pi}{2}-\frac{1}{4} r_{n}, \text { and } \\
& I_{k} \cap I_{j}=\emptyset \text { for } k<j .
\end{aligned}
$$

The first condition assures that we have the correct size and scaling of our figures, while the second condition assures that different diamonds do not intersect.

We proceed now to give the $n$-necklace $\mathcal{N}^{n}$ a metric measure structure. For $n \in \mathbb{N} \cup\{0\}$, endow $\mathcal{N}^{n}$ with the distance, $\mathrm{d}_{n}=\mathrm{d}_{L^{\infty}}$, induced from the $L^{\infty}$-norm in $\mathbb{R}^{2}$. To set a measure on the $n$-necklace we start by defining $\mathfrak{m}_{D_{n}} \ll \mathcal{L}^{2}$ on $D_{n}$ by

$$
\frac{\mathrm{dm}_{D_{n}}}{\mathrm{~d} \mathcal{L}^{2}}(x):=\left.\left[\frac{2}{9}\left(\frac{1}{4} r_{n}-\left|x-x_{n}\right|\right)\right]^{-1} \cos ^{2}(x) \chi\right|_{D_{n}}(x) \quad \text { for } n \in \mathbb{N},
$$

and $\left.\chi\right|_{A}$ the characteristic function of the set $A$. Denote by $\mathrm{D}^{n}=\cup_{1 \leq k \leq n} D_{k}$ the union of all diamonds $D_{k} \subset \mathcal{N}^{n}$, by $\mathrm{L}^{n}:=\mathcal{N}^{n} \backslash \mathrm{D}^{n}$ its complement, and we write $L^{0}:=\mathcal{N}^{0}$. We set on $\mathcal{N}^{n}$ the measure $\mathfrak{m}_{n}$ defined as

$$
\begin{aligned}
\mathrm{d} \mathfrak{m}_{n} & :=\mathrm{dm}_{\mathrm{D}^{n}}+\left.\cos ^{2}(x) \mathrm{d} \mathcal{H}^{1}\right|_{\mathrm{L}^{n}}, \quad \text { where } \\
\mathfrak{m}_{\mathrm{D}^{n}} & :=\sum_{1 \leq k \leq n} \mathfrak{m}_{D_{k}} .
\end{aligned}
$$

In words, the measure $\mathfrak{m}_{n}$ has a 2-dimensional contribution coming from $\mathrm{D}^{n}$, which has constant density for fixed $x$-coordinate, and a 1-dimensional contribution coming from $\mathrm{L}^{n}$, which is absolutely continuous w.r.t. the 1-dimensional Hausdorff measure. Finally, we 
define the fancy necklace as the measured Gromov-Hausdorff limit $\left(\mathcal{F} \mathcal{N}, \mathrm{d}_{\mathcal{F} \mathcal{N}}, \mathfrak{m}_{\mathcal{F N}}\right):=$ $\mathrm{mGH} \lim _{n \rightarrow \infty}\left(\mathcal{N}^{n}, \mathrm{~d}_{n}, \mathfrak{m}_{n}\right)$. Since in Lemma 5.4 we will show that $n$-necklaces satisfy the $\operatorname{MCP}(2,3)$-condition, for all $n \in \mathbb{N}$, the existence of the limit is guaranteed by the compactness of MCP-spaces.

It will be convenient to fix some notation before presenting our next Lemma. Given a sequence $\left\{\left(r_{i}, x_{i}\right)\right\}_{n \in \mathbb{N}}$ consider the $m$-necklace $\left(\mathcal{N}^{m}, \mathrm{~d}_{m}, \mathfrak{m}_{m}\right)$ constructed from it. We will call "projected $(m-1)$-necklace", denoted by $\left(P \mathcal{N}_{k}^{m-1}, \mathrm{~d}_{m-1}, \mathfrak{m}_{m-1}^{\prime}\right)$, the $(m-1)$-necklace constructed from the sequence $\left\{\left(r_{i}, x_{i}\right)\right\}_{i \neq k}$ for $1 \leq k \leq m$. That is, $P \mathcal{N}_{k}^{m-1}$ is the necklace with $(m-1)$ diamonds obtained by removing the $k$ th-diamond from $\mathcal{N}^{m}$. The $x$-coordinate of vertices of diamonds $D_{n}$ will be denoted by $x_{n}^{ \pm}=x_{n} \pm 1 / 4 r_{n}$, and for $x \in \mathcal{N}^{n}$ and $B \subset \mathcal{N}^{n}$, we define the height as $h(w, B):=\mathcal{H}^{1}(B \cap\{x=w\})$. Moreover, we define the following set of geodesics,

$$
\Upsilon\left(B_{0}, B_{1}\right):=\left\{\gamma \in \operatorname{Geo}\left(\mathcal{N}^{n}\right) \| \gamma \text { is a line segment with } \gamma_{i} \in B_{i}, i=0,1\right\} .
$$

The set $\Upsilon\left(B_{0}, B_{1}\right)$ consists of Euclidean geodesics that go from $B_{0}$ to $B_{1}$. (Take into consideration that there exist many non-Euclidean geodesics in $\left(\mathcal{N}^{m}, \mathrm{~d}_{m}, \mathfrak{m}_{m}\right)$.) Lastly, for $|y| \leq r_{k} / 36$ and $k \in \mathbb{N}$ define $\gamma^{k, y} \in \operatorname{Geo}\left(\mathcal{N}^{n}\right)$ as the geodesic obtained after gluing $\Upsilon\left(\left(x_{k}^{-}, 0\right),\left(x_{k}, y\right)\right)$ with $\Upsilon\left(\left(x_{k}, y\right),\left(x_{k}^{+}, 0\right)\right)$ and reparametrizing. The image of $\gamma^{k, y}$ is the union of a line segment going from the left vertex of $D_{k},\left(x_{k}^{-}, 0\right)$, to $\left(x_{k}, y\right)$ with its reflection over $\left\{x=x_{k}\right\}$. Define $M^{k}$ as the set of all such geodesics for $|y| \leq r_{k} / 36$.

We are ready to prove our next lemma.

Lemma 5.4 The $\mathrm{mms}\left(\mathcal{F} \mathcal{N}, \mathrm{d}_{\mathcal{F} \mathcal{N}}, \mathfrak{m}_{\mathcal{F N}}\right)$ satisfies the $\operatorname{MCP}(2,3)$-condition.

Proof The stability of the MCP-condition assures that it's enough to show that $\left(\mathcal{N}^{n}, \mathrm{~d}_{n}, \mathfrak{m}_{n}\right)$ $\in \operatorname{MCP}(2,3)$ for every $n \in \mathbb{N}$ and every sequence $\left\{\left(x_{k}, r_{k}\right)\right\}_{k \in \mathbb{N}} \subset \mathbb{R}^{2}$ that satisfies (5.3). Accordingly, we fix $n \in \mathbb{N}$ and such sequence. We proceed using key ideas from a proof in [23].

Definition 2.12 of the MCP condition requires that, for every $\tilde{z}=(\tilde{x}, \tilde{y})$ and $A \subset \mathcal{N}^{n}$ with $0<\mathfrak{m}_{n}(A)<\infty$, we give a measure $\pi \in \mathcal{P}\left(\operatorname{Geo}\left(\mathcal{N}^{n}\right)\right)$ such that $\left(\mathrm{e}_{0}\right)_{\#} \pi=\delta_{\tilde{z}}$, $\left(\mathrm{e}_{1}\right)_{\#} \pi=\left.\mathfrak{m}_{n}(A)^{-1} \mathfrak{m}_{n}\right|_{A}$, and inequality (2.13) is valid. Given $\tilde{z}$ and $A$ we will choose a set of geodesics $\Gamma=\Gamma_{\tilde{z}, A} \subset \operatorname{Geo}\left(\mathcal{N}^{n}\right)$ and define $\pi$ as the optimal geodesic plan arising from the lift of the induced to the optimal transport going along geodesics in $\Gamma$. However, we reduce before the number of transports that we need to study.

To begin with, note that we can analyze separately the sets $A_{x^{\prime}}=A \cap\left\{x=x^{\prime}\right\}$ for a fixed $x^{\prime}$. The simplification can be made because we will assure that the first coordinate contributes to the dilatation of the measure $\mathfrak{n}_{t}:=\left(\mathrm{e}_{t}\right)_{\#} \pi$ a factor equal to $t$. We will achieve this by picking geodesics with projection $\mathrm{p}_{1}(\gamma(t))=(1-t) \tilde{x}+t x^{\prime}$ for $\left(x^{\prime}, y^{\prime}\right)=z^{\prime} \in A$. Therefore the analysis reduces to estimating separately the dilatation of the sets $A_{x^{\prime}}$ for every $x^{\prime} \in \mathrm{p}_{1}(A)$. Accordingly, to verify the $\operatorname{MCP}(2,3)$-condition, it is enough to provide a set $\Gamma \subset \operatorname{Geo}\left(\mathcal{N}^{n}\right)$ such that $\mathrm{e}_{0}(\Gamma)=\tilde{z}, \mathrm{e}_{1}(\Gamma) \in A_{x^{\prime}}$, and

$$
\frac{\mathrm{dn}_{t}}{\mathrm{dm}_{n}}\left(\gamma_{t}\right) \leq \frac{\sin ^{2}(l(\gamma))}{t \sin ^{2}(t l(\gamma))} \frac{\mathrm{dn}_{1}}{\mathrm{dm}_{n}}\left(\gamma_{1}\right) \quad \text { for all } t \in[0,1], x^{\prime} \in \mathrm{p}_{1}(A), \gamma \in \Gamma .
$$

Claim 1 It's sufficient to check that $\left(\mathcal{N}^{m}, \mathrm{~d}_{m}, \mathfrak{m}_{m}\right) \in \operatorname{MCP}(2,3)$ for $m=0,1,2$. 
Proof First note that if $\tilde{z}, z^{\prime} \notin D_{k}$ for some $k \in\{1, \ldots, n\}$ then we can choose $\Gamma$ in a way that makes the density of $\frac{\mathrm{dn}_{t}}{\mathrm{dm}_{n}}$ independent of $y \in \mathrm{p}_{2}\left(D_{k}\right)$, that is, $\frac{\mathrm{dn}_{t}}{\mathrm{dm}_{n}}((x, y))=$ $\frac{\mathrm{dn}_{t}}{\mathrm{dm}_{n}}((x, 0))$ for $(x, y) \in D_{k}$. We can do this by choosing geodesics whose restriction to $D_{k}$ is exactly the set $M^{k}$. This choice of $\Gamma$ grants that the analysis of the transport of the measure inside $\left(\mathcal{N}^{n}, \mathrm{~d}_{n}, \mathfrak{m}_{n}\right)$ is equivalent to the analysis of the transport of the measure inside the projected $(n-1)$-necklace $\left(P \mathcal{N}_{k}^{n-1}, \mathrm{~d}_{n-1}, \mathfrak{m}_{n-1}^{\prime}\right)$. Furthermore, observe that if $n>2$ there exist at least $(n-2)$ such diamonds, $D_{k_{i}}$, for every $\tilde{z}, z^{\prime} \in \mathcal{N}^{n}$. Thus, for every transport inside $\mathcal{N}^{n}$ we can project at least $(n-2)$ times, reducing the task to checking the $\operatorname{MCP}(2,3)$-condition in the $m$-necklaces, for $m=0,1,2$.

In [33] and [23] it is shown that the 0-necklace and 1-necklace satisfy the $\operatorname{MCP}(2,3)$ condition, this covers the cases of $m=0,1$ so we move to $m=2 .{ }^{9}$ We assume, because of symmetry, that $\tilde{x} \leq x$ and fix $\tilde{z} \in \mathcal{N}^{2}$ and $A_{x^{\prime}} \subset \mathcal{N}^{2}$. We conclude from the preceding claim that the only situation left to check is that of $\tilde{x} \in D_{1}$ and $x^{\prime} \in D_{2}$. Let's first explain intuitively the way we transport the measure in this case. We start by expanding the measure uniformly from $\tilde{x}$ to a set $\hat{A}_{\hat{x}}$ with the same relative height as $A_{x^{\prime}}$. Then we transport the measure from $\hat{A}_{\hat{x}}$ to $x_{1}^{+}$without changing the relative height of the set $A_{t}:=\mathrm{e}_{t}(\Gamma)$ with respect to $D_{1} \cap\left\{x=\gamma_{t}\right\}$. We continue through $\mathrm{L}^{2}$ and expand again keeping the heights ratio constant from $x_{2}^{-}$to $A_{x^{\prime}}$. The image of a transporting geodesic is the union of segments of straight lines described below, see Fig. 1. In detail, to define $\Gamma$ first choose any set $\hat{A}_{\hat{x}} \subset$ $D_{1} \cap\{x=\hat{x}\}$ such that

$$
\frac{h\left(\hat{x}, D_{1}\right)}{h\left(\hat{x}, \hat{A}_{\hat{x}}\right)}=\frac{h\left(x^{\prime}, D_{2}\right)}{h\left(x^{\prime}, A_{x^{\prime}}\right)},
$$

for $\hat{x}=\frac{1}{5}\left(\frac{r_{1}}{4}+4\left(\tilde{x}-x_{1}\right)\right)+x_{1}$. Write $\hat{t}:=\frac{\hat{x}-\tilde{x}}{x-\tilde{x}}, t_{1}:=\frac{x_{1}^{+}-\tilde{x}}{x-\tilde{x}}$, and $t_{2}:=\frac{x_{2}^{-}-\tilde{x}}{x-\tilde{x}}$ for the times at which the $x$-coordinate of any geodesic $\gamma \in \Upsilon\left(\tilde{x}, A_{x^{\prime}}\right)$ is equal to $\hat{x}, x_{1}^{+}$, and $x_{2}^{-}$. Geodesics in $\Upsilon\left(\tilde{x}, D_{1} \cap\{x=\hat{x}\}\right)$ have the same length. Now define $\Gamma$ as the set of all geodesics satisfying the following: $\operatorname{rest}_{0}^{\hat{t}}(\gamma) \in \Upsilon\left(\hat{x}, \hat{A}_{\hat{x}}\right)$, rest ${ }_{\hat{t}}^{t_{1}}(\gamma) \in M^{1}$, rest $_{t_{1}}^{t_{2}}(\gamma) \in$ $\Upsilon\left(x_{1}^{+}, x_{2}^{-}\right)$, and rest $\left.t_{2}(\gamma) \in M^{2}\right|_{A_{x^{\prime}}}$, where $\left.M^{2}\right|_{A_{x^{\prime}}}$ is the subset of geodesics of $M^{2}$ that cross through $A_{x^{\prime}}$.

We now estimate the density of the corresponding measure, for $\gamma(t)=\left(x_{t}, y_{t}\right)$ we have that

$$
\frac{\mathrm{dn}}{\mathrm{d} \mathfrak{m}}\left(\gamma_{t}\right)=\frac{1}{t} \frac{h\left(x_{t}, D_{1}\right)}{h\left(x_{t}, A_{t}\right)}=\frac{1}{t^{2}} \frac{h\left(x_{t}, D_{1}\right)}{h\left(\hat{x}, \hat{A}_{\hat{x}}\right)} \frac{h\left(\hat{x}, D_{1}\right)}{h\left(\hat{x}, D_{1}\right)}=\frac{1}{t^{2}} \frac{h\left(x_{t}, D_{1}\right)}{h\left(x, D_{1}\right)} \frac{\mathrm{dn}_{1}}{\mathrm{~d} \mathfrak{m}}\left(\gamma_{1}\right),
$$

for $0 \leq t \leq \hat{t}$. The shape of the diamond $D_{1}$ allows to estimate $\frac{h\left(x_{t}, D_{1}\right)}{h\left(x, D_{1}\right)} \leq\left(\frac{5}{4}-\frac{t}{4}\right)$. We can bound the time when the geodesics reach $\hat{x}$ by $\hat{t} \leq r_{1} / 5 \leq 1 / 5$, and the length of the geodesics is necessarily $l \leq \pi / 2$. Moreover in [23] the estimate $\frac{5}{4}-\frac{t}{4} \leq t \frac{\sin ^{2}(d)}{\sin ^{2}(t d)}$ for all $(t, d) \in[0,1 / 5] \times(0, \pi / 2+1 / 4)$ is proved. Putting inequalities together we obtain inequality (5.5) for $t \in[0, \hat{t}]$.

To finish, note that for $t \in[\hat{t}, 1]$, the relative density of $\mathfrak{n}_{t}$ is independent of the $y$ coordinate. Thus, its density is equal to the one of the transport in the 0 -necklace, which is a $\operatorname{MCP}(2,3)$-space. This shows that inequality (5.5) is satisfied also for $t \in[\hat{t}, 1]$, hence, in the complete interval $t \in[0,1]$.

\footnotetext{
${ }^{9}$ More precisely, the proof in [23] can be repeated verbatim by doing minor modifications.
} 
Observe that the automorphism groups of $\left(\mathcal{F} \mathcal{N}, \mathrm{d}_{\mathcal{F N}}, \mathfrak{m}_{\mathcal{F N}}\right)$ are $\mathrm{G}=\Pi^{\infty}\{ \pm 1\}$. This proves Proposition 1.5 and confirms that the measure contraction property, without extra assumptions, does not guarantee smoothness of the automorphism groups.

Acknowledgements The author would like to express his gratitude to Jürgen Jost for his crucial support and valuable advice. He is also very grateful to Rostislav Matveev, and Jim Portegies for their essential and friendly involvement in this work.

He also thanks Fernando Galaz-Garcia, Yu Kitabeppu, Martin Kell, and Tapio Rajala for, among other things, discussions that led to Remark 4.6, and Fabio Cavalletti and Andrea Mondino for bringing [5] to his attention. Lastly, the author is thankful to Nidhi Kaihnsa, Niccolò Pederzani, and Ruijun Wu for commenting on earlier versions of the manuscript, and to the anonymous reviewers for their comments. This work was supported by the IMPRS program of the Max Planck Institute for Mathematics in the Sciences and partially by CONACYT. Open access funding provided by Max Planck Society.

Open Access This article is distributed under the terms of the Creative Commons Attribution 4.0 International License (http://creativecommons.org/licenses/by/4.0/), which permits unrestricted use, distribution, and reproduction in any medium, provided you give appropriate credit to the original author(s) and the source, provide a link to the Creative Commons license, and indicate if changes were made.

\section{References}

1. Ambrosio, L., Gigli, N., Mondino, A., Rajala, T.: Riemannian Ricci curvature lower bounds in metric measure spaces with $\sigma$-finite measure. Trans. Amer. Math Soc. 367(7), 4661-4701 (2015)

2. Ambrosio, L., Gigli, N., Savaré, G.: Metric measure spaces with Riemannian Ricci curvature bounded from below. Duke Math J. 163(7), 1405-1490, 05 (2014)

3. Burago, Y., Burago D., Ivanov, S.: A course in metric geometry, vol. 33 of Grad. Studies in Math. Americ. Math. Soc. (2001)

4. Cheeger, J., Colding, T.H.: On the structure of spaces with Ricci curvature bounded below II. J. Differ. Geom. 54(1), 13-35 (2000)

5. Cavalleti, F., Mondino, A.: Optimal maps in essentially non-branching spaces. (2016). arXiv:1609.00782. https://doi.org/10.1142/S0219199717500079. To appear in Comm. Cont. Math.

6. Colding, T.H., Naber, A.: Sharp Hölder continuity of tangent cones for spaces with a lower Ricci curvature bound and applications. Ann. Math. 176(2), 1173-1229 (2012)

7. Le Donne, E.: Metric spaces with unique tangents. Ann. Acad. Sci. Fenn. Math. 36, 683-694 (2011)

8. van Dantzig, D., van der Waerden, B.L.: Über metrisch homogene räume. Abh. Math. Semin. Univ. Hambg. 6(1), 367-376 (1928)

9. Erbar, M., Kuwada, K., Sturm, K.-T.: On the equivalence of the entropic curvature-dimension condition and Bochner's inequality on metric measure spaces. Invent. Math. 201(3), 993-1071 (2015)

10. Fukaya, K.: Theory of convergence for Riemannian orbifolds. Jpn. J. Math., New Series 12(1), 121-160 (1986)

11. Fukaya, K., Yamaguchi, T.: The fundamental groups of almost nonnegatively curved manifolds. Ann. Math. 136(2), 253-333 (1992)

12. Fukaya, K., Yamaguchi, T.: Isometry groups of singular spaces. Math. Z. 216(1), 31-44 (1994)

13. Gleason, A.M.: Groups without small subgroups. Ann. Math. 56(2), 193-212 (1952)

14. Gigli, N., Mondino, A., Rajala, T.: Euclidean spaces as weak tangents of infinitesimally Hilbertian metric measure spaces with Ricci curvature bounded below. J. Reine Angew. Math. 705, 233-244 (2015)

15. Gigli, N., Mondino, A., Savaré, G.: Convergence of pointed non-compact metric measure spaces and stability of Ricci curvature bounds and heat flows. Proc. Lond. Math. Soc. 111(5), 1071-1129 (2015)

16. Gigli, N., Rajala, T., Sturm, K.T.: Optimal maps and exponentiation on finite-dimensional spaces with Ricci curvature bounded from below. J. Geom. Anal. 26, 2914 (2016)

17. Guijarro, L., Santos-Rodriguez, J.: On the isometry group of RCD*(k,n)-spaces. (2016). arXiv:1608.06467

18. Harvey, J.: Equivariant Alexandrov geometry and orbifold finiteness. J. Geom. Anal. 26(3), 1925-1945 (2016)

19. Kell, M.: Sectional curvature-type conditions on metric spaces. ArXiv e-prints, arXiv:1601.03363 (2016) 
20. Ketterer, C.: Cones over metric measure spaces and the maximal diameter theorem. J. Math. Pures Appl. 103(5), 1228-1275 (2015). ISSN 0021-7824, https://doi.org/10.1016/j.matpur.2014.10.011

21. Kell, M., Mondino, A.: On the volume measure of non-smooth spaces with Ricci curvature bounded below. (2016). arXiv:1607.02036

22. Kobayashi, S., Nomizu, K.: Foundations of Differential Geometry, vol. I. Wiley, New York (1963)

23. Ketterer, C., Rajala, T.: Failure of topological rigidity results for the measure contraction property. Potential Anal. 42(3), 645-655 (2015)

24. Lott, J., Villani, C.: Ricci curvature for metric-measure spaces via optimal transport. Ann. Math. 169(3), 903-991 (2009)

25. Mondino, A., Naber, A.: Structure theory of metric-measure spaces with lower Ricci curvature bounds i. (2014). arXiv: 1405.2222

26. Myers, S.B., Steenrod, N.E.: The group of isometries of a Riemannian manifold. Ann. Math. 40(2), 400-416 (1939)

27. Ohta, S.: Examples of spaces with branching geodesics satisfying the curvature-dimension condition. Bull. Lond. Math. Soc. 46(1), 19-25 (2014)

28. Petrunin, A.: Alexandrov meets Lott-Villani-Sturm. Münster J. Math. 4, 53-64 (2011)

29. Rizzi, L.: Measure contraction properties of carnot groups. Calc. Var. 55(3), 1-20 (2016)

30. Rajala, T., Sturm, K.-T.: Non-branching geodesics and optimal maps in strong $\mathrm{CD}(\mathrm{k}, \infty)$-spaces. Calc. Var. 50(3), 831-846 (2014)

31. Sosa, G.: On symmetric transformations in metric measure geometry (Unpublished doctoral thesis). Max Planck Institute for Mathematics in the Sciences and University of Leipzig, Leipzig, Germany

32. Sturm, K.-T.: On the geometry of metric measure spaces. Acta Mathematica 196(1), 65-131 (2006)

33. Sturm, K.-T.: On the geometry of metric measure spaces II. Acta Mathematica 196(1), 133-177 (2006)

34. Tao, T.: Hilbert's fifth problem and related topics. Number 153 in Graduate Studies in Math. Amer. Math. Soc. (2014)

35. Yamabe, H.: A generalization of a theorem of Gleason. Ann. Math. 58(2), 351-365 (1953)

36. Yamaguchi, T.: Isometry groups of spaces with curvature bounded above. Math. Z. 232(2), 275-286 (1999) 